\title{
Reverse Engineering in Product MANUFACTURING: AN OVERVIEW
}

\author{
KUMAR A.; JAIN, P. K. \& PATHAK, P. M.
}

Abstract: Reverse engineering plays vital role in the branch of the mechanical design and manufacturing based industry. This technique has been widely recognized as an important technique in the product design cycle. In regular computerized manufacturing environment, the operation order usually starts from the product design and ends with machine operation to convert raw material into final product. It is often essential to reproduce a CAD model of existing part using any digitization techniques, when original drawings or documentation are not available and used for analysis and modifications are required to construct a improved product design. In reverse engineering approach the important steps involved, are characterizations of geometric models and related surface representations, segmentation and surface fitting of simple and free-form shapes, and creating accurate CAD models. The chapter presents review on reverse engineering methodology and its application areas related to product design development. The product re-design and research with reverse engineering will largely reduced the production period and costs in product manufacturing industries.

Key words: reverse engineering, scanning techniques, point cloud/STL data, $C A D / C A M / C A E$
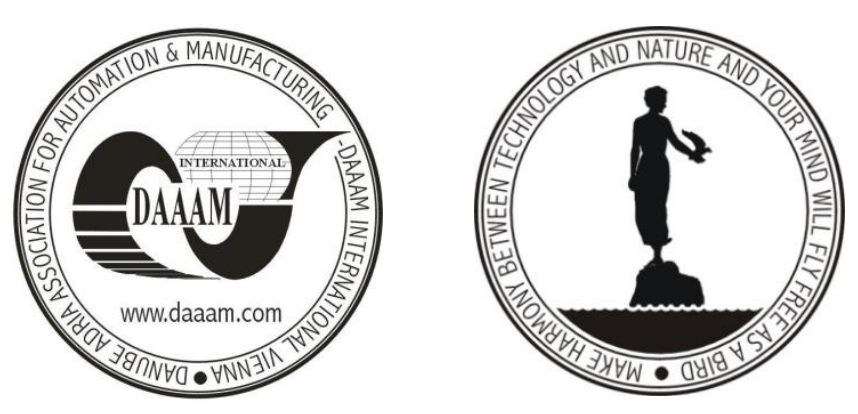

Authors' data: Kumar A[tul], Jain, P[ramod] K[umar]; Pathak, P[ushparaj] M[ani], Mechanical \& Industrial Engineering Department, Indian Institute of Technology, Roorkee, 247667, Uttarakhand India, atulkumar.iitr@gmail.com, pjainfme@iitr.ernet.in, pushppathak@gmail.com

This Publication has to be referred as: Kumar, A[tul]; Jain, P[ramod] K[umar] \& Pathak, P[ushparaj] M[ani] (2013) Reverse Engineering in Product Manufacturing: An Overview , Chapter 39 in DAAAM International Scientific Book 2013, pp. 665678, B. Katalinic \& Z. Tekic (Eds.), Published by DAAAM International, ISBN 9783-901509-94-0, ISSN 1726-9687, Vienna, Austria

DOI: $10.2507 /$ daaam.scibook.2013.39 
Kumar, A.; Jain, P. K. \& Pathak, P. M.: Reverse Engineering in Product Manufactu...

\section{Introduction}

The world has witnessed three digital convergences during the past three decades. Each time new technologies break down the barrier between physical and digital forms, new products and new markets has been created. The 1970s ushered in digitized sound using signal processing (1D), which made analog and digital conversion part of a common language in the telecom industry. The 1980s brought digitized fonts and pictures using image processing (2D). The convenience of switching between electronic and paper documents changed the publishing industry and the way to store and share information. The third convergence, beginning in the 1990s, focus on digitizing the physical world using geometry processing (3D). The convergence of physical and digital worlds enabled by reverse and forward engineering technologies should fundamentally change the way products are designed, manufactured, and marketed. By create a digital duplicate of world as easily as taking a digital picture, the biggest breakthrough of the twenty-first century will be in manufacturing industry.

Reverse engineering can be applied to re-create either the high-value commercial parts for business profits or the valueless legacy parts for historical restoration. To accomplish this task, the engineer needs an understanding of the functionality of the original part and the skills to replicate its characteristic details. In the fields of mechanical engineering and industrial manufacturing, reverse engineering refers to the method of creating engineering design and documentation data from existing parts and their assemblies. While in conventional engineering process, transforms engineering concepts and models into real parts, in the reverse engineering approach real parts are transformed into engineering models and concepts. Reverse engineering has a very common a broad range area such as mechanical engineering, software engineering, animation/entertainment industry, microchips, chemicals, electronics, pharmaceutical products etc. Focusing on the mechanical engineering domain, through the application of reverse engineering techniques an existing part is recreated by acquiring its' surface or geometrical features data using contact or non contact digitizing or measuring devices. By using reverse engineering, creation of product takes advantage of the extensive use of $\mathrm{CAD} / \mathrm{CAM} / \mathrm{CAE}$ systems. And apparently provides enormous gains in improving in quality, materials properties, efficiency of re-design, manufacture and analysis. Therefore, reverse engineering is going with substantial business benefits in shortening the product development cycle.

Reverse engineering has been used to produce many mechanical parts, such as seals, O-rings, bolts and nuts, gaskets, and engine parts, and is widely used in many industries (Tut, 2010). The Society of Manufacturing Engineers (SME) states that the practice of reverse engineering "starting with a finished product or process and working backward in logical fashion to discover the underlying new technology" (Francis, 1988). Manufacturers all over the world have practiced reverse engineering in their product development. The new analytical technologies, such as threedimensional (3D) laser scanning and high-resolution microscopy, have made reverse engineering easier, but there is still much more to be learned. Several professional 
organizations have provided the definitions of reverse engineering from their perspectives. It has been incorporates in appropriate mechanical design and manufacturing engineering standards and multiple realistic product constraints with broad knowledge in multiple disciplines such as:

- Applying knowledge of mathematics, engineering, and science in data analysis and interpretation.

- Using techniques, instruments, and tools in reverse engineering applications

- Conducting appropriate experiments and tests to obtain the necessary data in reverse engineering.

- Identifying, formulating, and solving issues related to reverse engineering.

- Understanding legal and ethical responsibilities pertinent to reverse engineering.

- Assessing and evaluating documents and fostering attainment of objectives of a reverse engineering project.

The part produced through reverse engineering should be in compliance with the requirements contained in applicable program criteria. To achieve a successful reverse engineering process requires. Though it roots back to ancient times in history, the recent advancement in reverse engineering has elevated this technology to one of the primary methodologies utilized in many industries, including aerospace, automotive, consumer electronics, medical device, sports equipment, toy, and jewellery. It is also applied in forensic science and accident investigations.

\section{History}

Reverse engineering was often used during the Second World War and the Cold War. It is often used by military in order to copy other nation's technology, devices or information, or parts of which, have been obtained by regular troops in the fields or by intelligence operations. In the last few years, increased computational power, more computer memory, and high-speed contact or non-contact scanning devices, discrete geometry has gained increasing importance in automotive design, manufacturing, and quality assurance. In recent year, the impact of reverse engineering in manufacturing industry is increase day per day and it also plays a significant role in promoting industrial evolution by just introducing the expensive products and stimulating additional competition. However, the average life cycle of modern inventions is much shorter. To accommodate this rapid rate of reinvention of modern machinery and instruments, reverse engineering provides a high-tech tool to speed up the reinvention process for future industrial evolution. Reverse engineering plays a significant role in the aviation industry primarily because of the following reasons: maturity of the industry, advancement of modern technologies, and market demands. From the dawn of the aviation industry in the early 1900s to its hardware maturity with the development of jet aircraft in the 1950s, the aviation industry revolutionized the modes of transportation in about 50 years. 
Kumar, A.; Jain, P. K. \& Pathak, P. M.: Reverse Engineering in Product Manufactu...

\section{Reverse Engineering}

Reverse engineering is the process of obtaining a geometric CAD model from measurements acquired by contact or non-contact scanning technique of an existing physical model (Liang \& Grier, 2000). The characterizes typical procedure of reverse engineering is showing in figure 1.

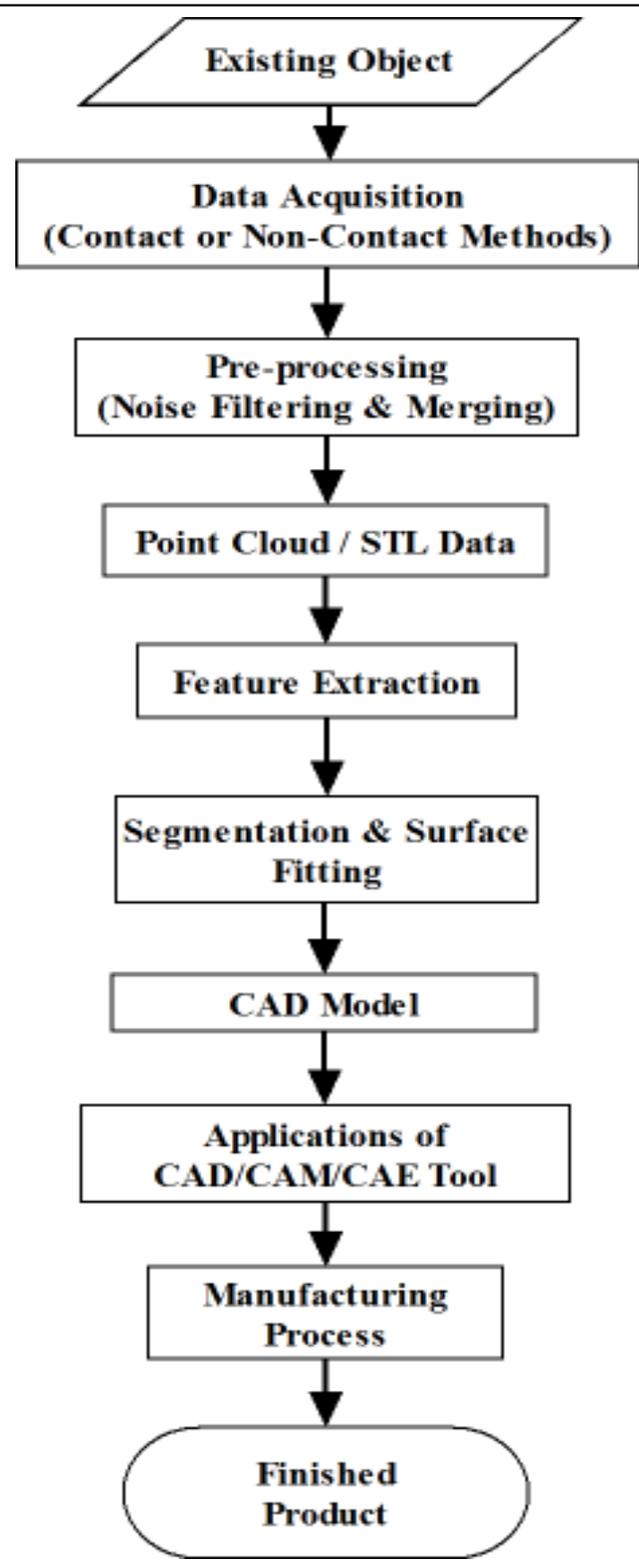

Fig. 1. Basic flow of reverse engineering

It consists of following steps: data acquisition, pre-processing (noise filtering and merging), triangulation, feature extraction, segmentation and surface fitting and application of CAD/CAM/CAE tools (Bidanda \& hosni, 1994; Chang \& Park, 1994). 


\section{Computer Aided Reverse Engineering}

Reverse Engineering originally emerged as the answer to provide spares for replacing broken or worn out parts for which no technical data was available. This can be the case if the part was originally imported (without drawings) or the drawings being misplaced or lost. Reengineering or reverse engineering such parts can be a less expensive option compared to re-importing, not only for immediate replacement, but also to create additional spares to maintain the product over a longer period. Computer-based surface models are indispensable in several fields of science and engineering. For example, the design and manufacturing of vehicles, such as cars and aircrafts, would not be possible without sophisticated CAD and simulation tools predicting the behaviour of the product. The point cloud acquisition generally is performed by stationary scanning devices, like laser-range or computer-tomography scanners. After taking multiple scans from various sides or by rotating the object, the sampled points are combined into a single point cloud, from which the surface needs to be reconstructed. The resulting adaptive reconstruction method is based upon the repetitive application of the following steps (Pal et al., 2005):

- Starting from an initial bounding point enclosing the original point cloud the hierarchical space partitioning creates a point set by recursively subdividing each individual point into sub-point

- The resulting mesh is obtained by subdividing the coarser mesh and adapting its topology at locations where point have been removed

- The final data mapping locally constrains the mesh toward the point cloud. All vertices are projected onto local tangent planes defined by the individual points.

Reverse Engineering has been defined as a process for obtaining the technical data of a critical spare component. Computer-aided reverse engineering relies on the use of computer-aided tools for obtaining the part geometry, identifying its material, improving the design, tooling fabrication, manufacturing planning and physical realization. A solid model of the part is backbone for computer-aided reverse engineering. The model data can be exported or imported into CAD/CAE/CAM systems using standard formats such as IGES, STL, VDA and STEP.

\section{Feature Based Reverse Engineering}

Feature-based models have been suitable for manufacturing the mechanical parts with reverse engineering. Also, feature-based models are ideal for industrial design and manufacturing since the model produced can be easily modified. Feature-based and constraint-based methods can be characterized as knowledge-based methods. As researchers, it is useful to exploit design intent and feature relationships that exist in models created for industrial use, because they justify some of the attributes of the object that are obsolete. Such information can be expressed by geometric constraints (Thompson et al. 1999). 
Kumar, A.; Jain, P. K. \& Pathak, P. M.: Reverse Engineering in Product Manufactu...

\section{Basic Steps in Reverse engineering}

As indicated earlier the main purpose of reverse engineering is to convert a discrete data set into a piecewise smooth, continuous model. In this section various aspects of this conversion are described. The discrete data set typically consists of (x; $\mathrm{y} ; \mathrm{z}$ ) coordinate values of measured data points. Concerning the organization of data with follow the following steps are (Várady et al., 1997):

\subsection{The Geometry Part Digitization}

The first objective of reverse engineering methodology is to digitize the physical model. Digitization is the process of capturing the data of the physical model and converting digital form. It can be achieved by utilizing either contact probing or noncontact sensing techniques. In figure 2 shows the classification of the acquiring 3D data into contact and non-contact methods. After taking multiple scans from various sides or by rotating the object, the sampled points are combined into a single point cloud, from which the surface needs to be reconstructed. The resulting adaptive reconstruction method is based upon the repetitive application of the following steps:

- Starting from an initial bounding point enclosing the original point cloud the hierarchical space partitioning creates a point set by recursively subdividing each individual point into sub-point

- The resulting mesh is obtained by subdividing the coarser mesh.

- The final data mapping locally constrains the mesh toward the point cloud.

- All vertices are projected onto local tangent planes defined by the individual points.

\subsection{Purpose of Object Surface Acquisition}

The measurement point group and STL data are used in two ways:

- Analyze own and other product (design)

- Confirm the accuracy of own products (Inspection)

Design purpose further divided into:

- Generate a 3D mockup to shorten the development period

- 3D data are not available, use as CAD data to implement analysis.

Inspection purpose is further divided into:

- Inspect object dimension

- Check the amount of deform material

- Define the life of object based on result. 


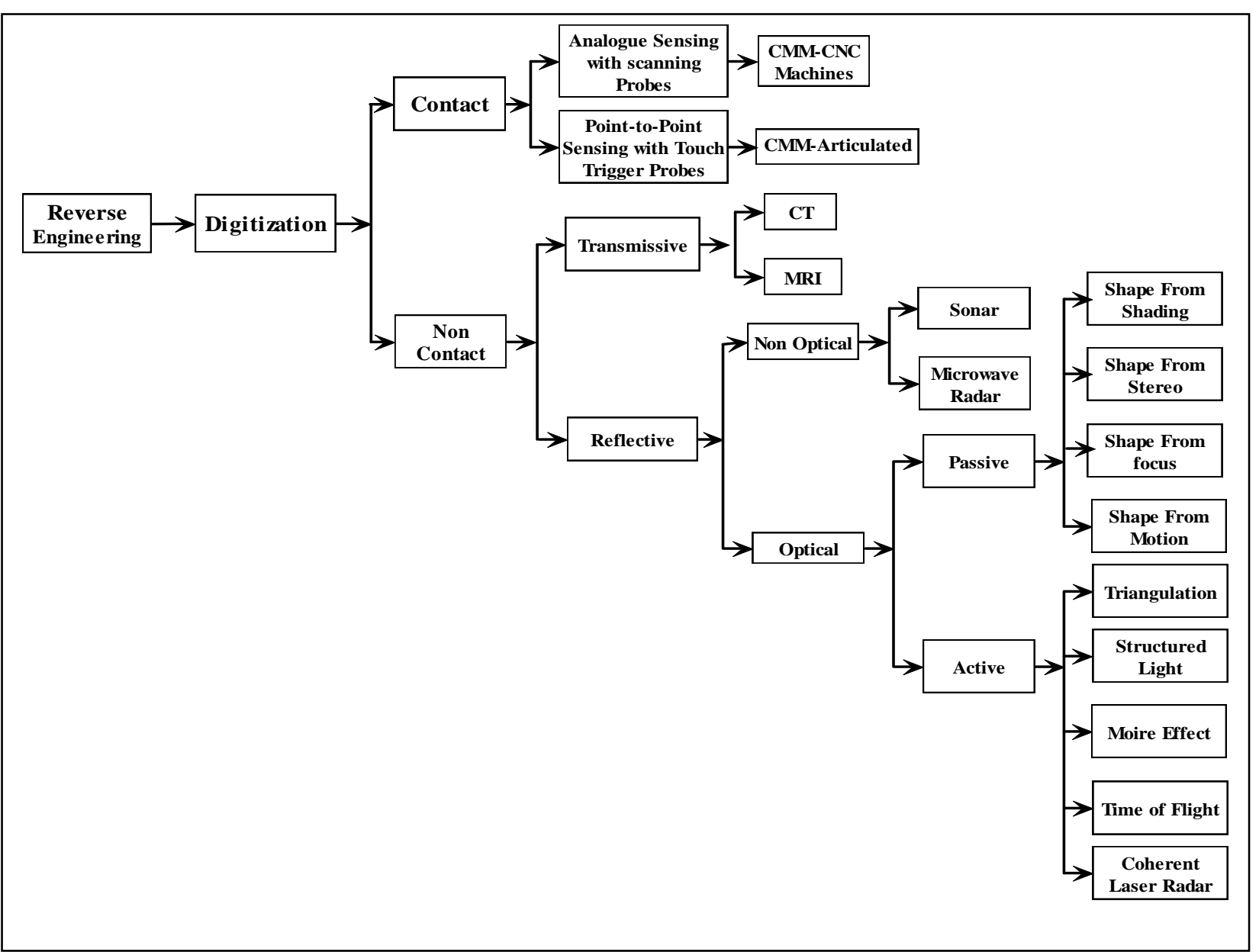

Fig. 2. Classifications of data acquisition techniques used in contact and non contact approaches for Revere Engineering systems (Kumar et al., 2012)

\subsection{Post-Processing}

Based on the past research on some curve smoothing theories, the vertices of the mesh are repositioned by computing the centroid of the directly connected neighbor vertices. To improve the quality of the generated mesh can be performed an additional optimization step. In a successive step these centroid are again predictable onto the tangent planes of the corresponding data sets according to define theories. Generally, mesh-optimization is a repetitive process, applied several times to obtain the most possible accuracy in surface quality which is help for 3D CAD model.

\subsection{Triangulation}

Based on past research on triangulation under reverse engineering environment, the mathematic theory and computational algorithms for triangulation have been well developed. The triangular polygon mesh with sufficient geometrical information can be created efficiently for a given set of data points. The fundamental concept in triangulation is Delaunay triangulation. In addition to Delaunay triangulation, there are several mathematic algorithms for triangulation, including marching cubes, Poisson surface reconstruction, moving least squares methods etc. While, some triangulation algorithms may not perfect as per requirements. They have been tending to generate meshes with a high triangle count. In addition, these algorithms implicitly 
Kumar, A.; Jain, P. K. \& Pathak, P. M.: Reverse Engineering in Product Manufactu... assume topology of the shape to be reconstructed from triangulation, and the parameters setting often influence results and stability.

\subsection{Segmentation}

One of the most important steps in reverse engineering is mesh segmentation. Segmentation is a complex process in which the original data points are subsets of each individual logically belongs to a primitive surface. Some more efficient noniterative segmentation methods are using and they are called direct segmentation methods. In general, the segmentation process is involves an estimate of first- and second-order surface properties. The first-order segmentation, which is based on normal vectors, provides an initial subdivision of the surface and detects sharp edges as well as flat or highly curved areas. The second-order segmentation subdivides the surface according to principal curvatures and provides a sufficient foundation for the classification of simple algebraic surfaces. Most of the segmentation algorithms come with surface fitting, which fits a best primitive surface of appropriate type to each segmented region. It is important to specify a hierarchy of surface types in the order of geometric complexity (Várady et al., 1997).

As discussed above, feature-based segmentation provides a sufficient foundation for the classification of primary and secondary geometry shown in figure 3 or parametric and non-parametric surfaces. Algebraic surfaces, such as planes, such as sphere, cylinders, and cones, and tori, are readily to be fitted to such regions.

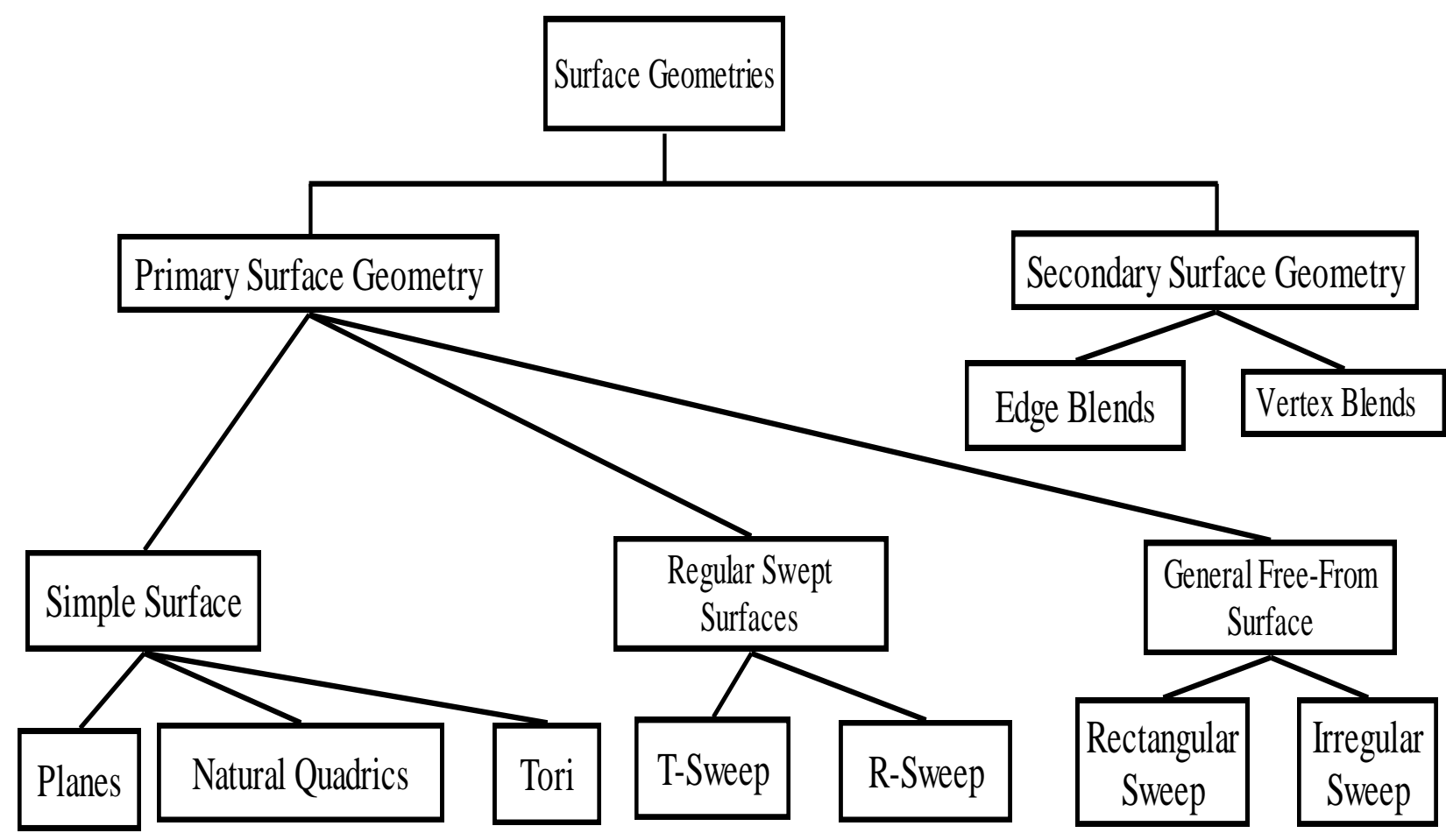

Fig. 3. Surface hierarchy 
In addition to primitive non parametric surfaces with a simple kinematic generation, such as sweep surfaces, revolved surfaces, extrusion surfaces, pipe surfaces, are directly compatible to CAD models.

\subsection{Solid Modeling}

Solid modeling is probably use for shape engineering process in support of reverse engineering using any modeling software such as Auto CAD, CATIA, Pro/E etc. There are two basic representations for solid models are boundary representation and feature-based representation. There have some methods, such as (Várady et al., 1998), proposed to automatically construct boundary representation models from point clouds or triangular mesh with profile curve. It is creating by connecting the point to point in spline manner. Some focused on manufacturing feature recognition for process planning purpose. However, none of the method is able to fully automate the construction process and generate fully parametric solid models.

\subsection{Solid Model Export}

Re-constructed the 3D model using reverse engineering, the software will have to exported the conventional CAD packages for supporting the design engineering aspect. The conventional solid model exchanges via standards, IGES or STEP, STL, are inadequate since parametric information, sketch constraints and dimensions, including solid features, feature tree, are completely not same through the exchanges. Direct solid model can be exported or imported in some software, such as liveTransfer ${ }^{\mathrm{TM}}$ module of Rapidform XOR3 with CAD/CAE/CAM systems using standard formats such as IGES, STL, VDA and STEP.

\section{Applications of Reverse Engineering}

Reverse engineering is a multidisciplinary approach and virtually can be applied to industrial field universally. The prime applications of reverse engineering are either to re-create a copy of part of the original part or retrace the events of what happened. It is widely used in software and information technology industries, from software code development to Internet network security. Thousands of parts are reinvented every year using reverse engineering to satisfy the aftermarket demands that are worth billions of dollars. The invention of digital technology has fundamentally revolutionized it. Compared to the aviation and automobile industries, the applications of digitalized reverse engineering in the life science and medical device industries have faced more challenges and advanced at a more moderate pace. However, some briefly description has been presented with reverse engineering applications as follows:

\subsection{In Mechanical Industry}

The term engineering is generally used to describe the act of creating something beneficial. Reverse engineering has been associated with the copying an original 
Kumar, A.; Jain, P. K. \& Pathak, P. M.: Reverse Engineering in Product Manufactu... design for competitive purposes. In the manufacturing world today, however, the concept of reverse engineering is being legally applied for producing new products or variations of old products. The term reverse comes from the concept of bi-directional data exchange between the digital and physical worlds. The primary thrust in the early development of computer-aided design (CAD), engineering (CAE) and manufacturing (CAM) was to create a product in a computer and bring the results out to the real world. CAD was supposed to be able to define a simple part or a complex assembly entirely from its dimensional characteristics. CAE components, such as structural or thermal analysis software, would take this digital representation and analyze it. The CAM software would take this same electronic definition and create the paths to cut the tools for part manufacture. Today, the reverse engineering is applying in surface creation of complex geometry mechanical parts such as turbine blade, gear, car engine, casing, gas kit etc.

\subsection{In Aerospace and Ship Hull Craft}

Reverse engineering approach has been used by Boeing and other aerospace companies to create digital inventories of spare parts or to convert legacy data into today's CAD environments. Reverse engineering method is a key to the future of aerospace manufacturing as CAD tool. The modern aerospace industry uses reverse engineering for these key reasons (Ping, 2008):

- To create legacy parts that does not have CAD models

- To overcome obstacles in data exchange

- To short out problems arising from discrepancies between the CAD master model and the actual tooling or as-built part

- To confirm the quality and performance by computer-aided inspection and engineering analysis

An industrial application of CAD is presented, which concerns the measurement and reengineering of the shape of a complete ship hull and of ship's parts, which is a frequently recurring task in the shipbuilding and ship repair sector. In order to choose the most appropriate measurement method, several typical aspects of our object of measurement, such as its size, possible obstructions and poor accessibility, have to be taken into consideration (Koelman, 2010).

\subsection{Software Industry}

Software reverse engineering is concerned with the analysis of an existing system The IEEE Standard for Software Maintenance (IEEE Std 1219-1993) defines reverse engineering as "the process of extracting software system information from source code." Generally speaking, the output of a reverse engineering activity is synthesized, higher-level information that enables the reverse engineer to better reason about the system and to evolve it in an effective manner. 
The process of reverse engineering typically starts with lower levels of information such as the system's source code, possibly also including the system's build environment. When conducting a reverse engineering activity, the reverse engineer follows a certain process. The workflow of the reverse engineering process can be decomposed into three subtasks extraction, analysis, and visualization. In practice, process has elements that make it both ad hoc and creative (Holger M. et al., 2012)

\subsection{In Medical Life Science}

The engineering originality of the human body has put reverse engineering in a unique place in the life science and medical device industries, particularly in implementing artificial parts into the human body. Applying scanned images with finite element analysis in reverse engineering helps engineers in precisely modeling customized parts that best fit individual patients. The baseline requirements for reverse engineering is in life science and medical devices for physiological characteristic of living cells, human organs, and the communications among them. Engineers and scientists often work in the reverse direction can be help by observed body behaviors and the biological elements there must underlie the mechanisms that can reproduce these biological functions.

Under reverse engineer environment the engineers, first have to identify the materials that are used for this part and characteristics medical device, then the part geometric form has to be accurately digitized, and the manufacturing process has to be verified. Reverse engineering is used in several medical fields: dentistry, hearing aids, artificial knees, and heart (Fu, 2008).

Based on requirement, advanced computer-aided manufacturing processes can build customized orthodontic devices for individual patients. The growth of reverse engineering applications is mostly dependent on technology evolution to make the wireless hearing aid smaller, more sophisticated, and more efficient at lower cost. The applications of reverse engineering to orthopedics, such as the knee, hip, or spine implantation, are very challenging, partially due to the complex motions of the knees, hips, or spine. A proper function of these implants manufactured by reverse engineering requires them to sustain multiaxial statistic stresses and various modes of dynamic loads.

It is also used to reconstruct the events just before and immediately after accidents in the aviation, automobile, and other transportation industries. Other fields, such as in fashion Design, in chemical industry, architecture and civil engineering, and art galleries, also find a lot of reverse engineering applications.

\section{A Short Review On Modern Digitization System}

The terms digitizing and scanning are often used to describe the same process. Traditionally, the term digitizing referred to the process of taking discrete points from a surface using a touch-trigger probe or optical techniques. The digital points cloud 
Kumar, A.; Jain, P. K. \& Pathak, P. M.: Reverse Engineering in Product Manufactu... could be captured from different digitization methods. A short review on scanning system has been described in table 1 (Sokovic \& Kopac, 2006):

\begin{tabular}{|c|c|c|}
\hline $\begin{array}{l}\text { Type of } \\
\text { Scanning }\end{array}$ & Pros & Cons \\
\hline $\begin{array}{l}\text { CCD } \\
\text { cameras }\end{array}$ & $\begin{array}{l}\text { It is very fast and possible to use } \\
\text { two or three cameras } \\
\text { simultaneously. With light } \\
\text { controlled. Non-contact method; } \\
\text { it is possible to scan of soft } \\
\text { Materials. In the case of special } \\
\text { coaxial lightening it is possible } \\
\text { to scan small diameters and high } \\
\text { depths in the Z-axis. It is } \\
\text { possible to scan very small } \\
\text { areas, } 1 \mathrm{~mm}^{2} \text { accuracy is about } \\
\text { some micrometers. In the case } \\
\text { of oily of wet parts scanning the } \\
\text { results are disfigured }\end{array}$ & $\begin{array}{l}\text { High price of equipment Accuracy } \\
\text { decreases linear depend of the } \\
\text { camera distance. Scanning angle is } \\
\text { equal regardless of the shape of the } \\
\text { parts surface. By scanning with } \\
\text { camera very high sharpness of visible } \\
\text { picture is required simultaneous } \\
\text { scanning of close and far surface } \\
\text { details demonstrates linear deviation } \\
\text { of the results respect to different } \\
\text { focus distance. Dust causes fault by } \\
\text { scanning }\end{array}$ \\
\hline Laser & $\begin{array}{l}\text { Precise and fast scanning in Z- } \\
\text { axis }(0.001 \mathrm{~mm} \text { or better) } \\
\text { Non-contact method } \\
\text { It is not possible to scan } \\
\text { reflective materials }\end{array}$ & $\begin{array}{l}\text { Very high price of equipment and It } \\
\text { is possible to scan of soft materials } \\
\text { and scanning in X-and Y-axis is } \\
\text { upto } 0.035-0.060 \mathrm{~mm} \text {. It is not } \\
\text { possible to scan on the area of } \\
\text { notches or steep surfaces because of } \\
\text { additional reflection and sensitivity } \\
\text { to draught and dust in the air }\end{array}$ \\
\hline Contact & $\begin{array}{l}\text { Very precise in all axis (depends } \\
\text { of the scanning equipment). } \\
\text { Very fast scanning of } \\
\text { geometrical well-known parts. } \\
\text { Very precise scanning of coins } \\
\text { and similar relief. Possibility of } \\
\text { manual or automatic scanning. } \\
\text { Hand scanning equipment is } \\
\text { useful for scanning very big } \\
\text { products such as: airplanes, } \\
\text { ships, big machines and devices. }\end{array}$ & $\begin{array}{l}\text { It is not appropriate to soft materials } \\
\text { Scanning of unknown surfaces is not } \\
\text { makes possible } \\
\text { or process of scanning is very slow } \\
\text { and inaccurate }\end{array}$ \\
\hline
\end{tabular}

Tab.1. Shows the pros and cons of scanning systems

\section{Reverse Engineering in Modern Industries}

The distinction between an original equipment manufacturer and a supplier has been blurred in recent years in today's dynamic and competitive global market. The 
application of reverse engineering, engine spare parts reproduced for the repair and replacement of worn-out components will have significant economic impact on the aviation industry and its customers. In the 1970s, to reverse engineer application for high pressure turbine blade was a challenge due to the need to decode highly guarded industry proprietary information. In the 2000 s, have technical innovations changed the reverse engineering process, also the practice itself is also more widely accepted. The production of quality reverse engineered parts does require the full reinvention of engineering design and manufacturing process. To obtain precise geometric information for the after market automobile parts, many companies also resort to the technology of digital scanning and reverse engineering. It provides a variety of auto parts, including spoilers, running boards, fenders, and wheel covers. The companies are not always able to take advantage of equipment manufacturer original CAD data, partially because the as-built parts are often slightly different from the CAD data.

The U.S. federal government, the reverse engineered automotive parts are certified by the industry itself. Automotive Parts Association encourages price and quality competition in the marketplace so that customer expenses are reduced but till maintaining part quality. One of the widely cited reverse engineering examples in the military is the Soviet Tupolve Tu-4 (Bull) bomber. During World War II, three battledamaged U.S. B-29 Super fortress bombers made emergency landings in the Soviet Union territory after missions to Japan. Reverse engineered projects are not successful very time. For example, a reproduction of the 1903 Wright Flyer fell into a puddle after attempting flight on December 15, 2003.This ill-fated flight attempt brought out another risk factor in reverse engineering. Even though, might have produced a seemingly identical replica of the original part, the operability of the reverse engineered part also depends on the operating environment (Wego, 2011). A successful reverse engineering program requires great attention to the miniature details and accuracy of all measurements, in addition to a thorough understanding of the functionality of the original part.

\section{Conclusion}

The fundamental principles and basic limitations of reverse engineering are similar in most industries. The general practice of reverse engineering, such as data collection, detailed analysis at a micro scale, modeling, prototyping, performance evaluation, and regulation compliance, are the same in principle for all industries. The success of this endeavor is usually subject to the general limitations of modern technologies. However, the specific methodologies used in different fields can be vastly different.

The engineering design supported by $\mathrm{CAD} / \mathrm{CAE} / \mathrm{CAM}$ techniques allows optimizing the product manufacturing with assistance of $\mathrm{CNC}$ machine, in management for rapid product development and rapid set-up production in advance to full file the customer demand of time. For some product development processes reverse engineering (RE) allows to generate surface models by three-dimensional (3D)-scanning technique, and consequently this approach must be permits to redesign 
Kumar, A.; Jain, P. K. \& Pathak, P. M.: Reverse Engineering in Product Manufactu...

and manufacture different parts (for cars, for household appliances) and tools (moulds, dies, press tools) in a short development period. As a result application of reverse engineering will gain speed for product realization system and largely decreases the manufacturing cost.

\section{References}

Bopaya, B. I. \& Hosni Y. A. (1994). Reverse Engineering and Its Relevance to Industrial Engineering: A Critical Review, International journal of Computers and Engineering, Vol. 26, No. 2, (1994), pp. 343-348

Fu, P. (2008). In Reverse engineering: An industry perspective, Springer, ed. V. Raja and K. J. Fernandes, 177-193. Berlin

Herbert J. Koelman (2010). Application of a photogrammetry-based system to measure and re-engineer ship hulls and ship parts: An industrial practices-based report, Computer-Aided Design, Vol. 42, (February, 2010), pp. 731-743

Holger M. Kienle and Johan Kraftand Hausi A. Müller (2012). Reverse EngineeringRecent Advances and Application, Software Reverse Engineering In The Domain Of Complex Embedded Systems, ISBN 978-953-51-0158-1, Janeza Trdine 9, 51000 Rijeka, Croatia

Kumar, A., Jain, P. K. and Pathak, P. M. (2012). Industrial Application of Point Cloud / Stl Data for Reverse Engineering, DAAAM International Scientific Book 2012, B. Katalinic (Ed.), Published by DAAAM International, ISBN 978-3-90150986-5, ISSN 1726-9687, Vienna, Austria. pp. 445-462

Pal, D. K., Ravi B. L., Bhargava S. Chandrasekhar, U. (2005). Computer-Aided Reverse Engineering for Rapid Replacement Parts: A Case Study Defence Science Journal, DESSIDOC, DRDO, New Delhi, pp. 1-14

Sokovic, M. \& Kopac, J. (2006). RE (reverse engineering) as necessary phase by rapid product development, Journal of Materials Processing Technology,Vol. 175, (2006), pp. 398-403

Thompson William B., Jonathan C. Owen, H. James de St. Germain, Stevan R. Stark, Jr., and Thomas C. Henderson. (1999). Feature-Based Reverse Engineering of Mechanical Parts, IEEE transactions on robotics and automation,Vol. 1, No. 15, (February, 1999), pp. 57-66

Tut V., A. Tulcan, C.Cosma, and Serban I. (2010). Application of CAD/CAM/FEA, Reverse Engineering And Rapid Prototyping In Manufacturing Industry, International Journal Of Mechanics, Vol. 4, No. 4, (2010), pp. 79-86

Varady; Tamas, Martin, Ralph R. \& Coxt, Jordan (1997). Reverse engineering of geometric models-an introduction, International Journal of Computer-Aided Design. Vol. 29, No. 4, (May, 1997), pp. 255-268

W. Wego, (2011). Reverse Engineering Technology of Reinvention, Taylor and Francis Group, LLC International Standard Book Number-13: 978-1-4398-0631-9 